\title{
A novel variant in fibrillin-1 is responsible for early-onset familial thoracic aortic aneurysms in Marfan patients
}

\author{
Yanyu Duan ${ }^{1,2,3}$, Haiying Chang ${ }^{1,2}$, Jiayuan Ling ${ }^{1,2}$, Shaoqiang Liu ${ }^{4}$, Yiming Zhong ${ }^{1,2,3}$ \\ ${ }^{1}$ Key Laboratory of Prevention and Treatment of Cardiovascular and Cerebrovascular Diseases, Ministry of Education, Gannan Medical University, \\ Ganzhou, China; ${ }^{2}$ Heart Medical Centre, First Affiliated Hospital of Gannan Medical University, Ganzhou, China; ${ }^{3}$ Ganzhou Technology Innovation \\ Center for Prevention and Treatment of Rare Cardiovascular Diseases, Ganzhou, China; ${ }^{4}$ Radiology Department, First Affiliated Hospital of Gannan \\ Medical University, Ganzhou, China \\ Contributions: (I) Conception and design: Y Duan; (II) Administrative support: Y Duan, Y Zhong; (III) Provision of study materials or patients: H \\ Chang, S Liu; (IV) Collection and assembly of data: Y Duan, J Ling; (V) Data analysis and interpretation: Y Duan; (VI) Manuscript writing: All \\ authors; (VII) Final approval of manuscript: All authors. \\ Correspondence to: Yiming Zhong. Key Laboratory of Prevention and Treatment of Cardiovascular and Cerebrovascular Diseases, Ministry of \\ Education, Gannan Medical University, Ganzhou, China. Email: yimingdoc@126.com.
}

\begin{abstract}
Background: Marfan syndrome (MFS) is an inherited connective tissue disorder that affects the skeletal, ocular, and cardiovascular system. The disease's severity and clinical manifestations vary greatly due to pathogenic variants which, combined with a lack of research on the correlation between MFS's genotype and phenotype, make MFS a challenging disease to diagnose. This study aims to further the understanding of MFS by shedding light on the clinical manifestation of a novel variant in fibrillin-1 (FBN1) - the protein responsible for the genetic defects that lead to MFS.
\end{abstract}

Methods: A patient was diagnosed with MFS by combining a clinical examination (based on the 2010 revision to Ghent nosology criteria) with a targeted next-generation sequence analysis. The functional analysis of the causal mutation and the clinical details of the affected patient were then analyzed.

Results: The FBN1 heterozygous variant c.5081_5082insT, which is known to delete large fragments from amino acids 1702 to 2871, was found in the proband patient and her son. The two also displayed the skeletal and cardiovascular manifestations of MFS. In addition, the 14-year-old son was identified as having a dilated aortic bulb at the same rupture site of the proband's dissection, and the proband's mother also died at age 32 due to aortic dissection.

Conclusions: The FBN1 variant c.5081_5082insT (p.Leu1694fs*9) is a pathogenic mutation that can cause MFS patients to experience early-onset familial thoracic aortic aneurysms (TAA). We hope that this discovery can provide further insight into the treatment of MFS patients with truncating variants in exons 42-65.

Keywords: Thoracic aortic aneurysm/dissection; frameshift mutation; fibrillin-1 (FBN1); Marfan syndrome (MFS)

Submitted May 27, 2021. Accepted for publication Jul 07, 2021.

doi: 10.21037/atm-21-3104

View this article at: https://dx.doi.org/10.21037/atm-21-3104

\section{Introduction}

Marfan syndrome (MFS) is a connective tissue disorder with pleiotropic manifestations involving the cardiovascular, ocular, skeletal, skin, lung, and central nervous systems. The disease's diagnosis is based primarily on the clinical interaction of these systems as outlined in the Ghent nosology criteria. Mortality due to the disease is mainly associated with the development of cardiovascular events, such as heart failure, aortic aneurysm, and subsequent aortic dissection (1).

More than $90 \%$ of MFS cases are caused by pathogenic 
heterozygous variants which are encoded in the extracellular matrix glycoprotein fibrillin-1 (FBN1). Previous studies have been explored the relationship between the location and type of FBN1 mutations and the clinical outcomes of MFS patients (2). However, these efforts have been proven to be difficult by the known inter-familial and intra-familial clinical variability in MFS patients (3). Furthermore, mutations in FBN1 lead to different clinical disorders, such as non-syndromic familial thoracic aortic aneurysms, familial kyphoscoliosis, adolescent idiopathic scoliosis, and Weill-Marchesani syndrome et al. (4). FBN1 perform important biological functions as a calciumbinding microfibrillar structural molecule and a regulator of transforming growth factor $\beta$ (TGF- $\beta$ ) signaling (5), beyond serving as scaffolds for elastin deposition. In this manner, the tissue-specific fibrillin microfibril network structure cooperated with biological functions to force influence on tissue growth and homeostasis (4). This indicates that more studies on genotype-phenotype correlation are warranted.

In this study we have sought to identify and determine how a novel variant in FBN1 might be responsible for early-onset familial thoracic aortic aneurysms (TAA) in MFS patients. We believe such research can contribute to improving the diagnosis and treatment of MFS patients with truncating variants in exons 42-65.

We present the following article in accordance with the MDAR reporting checklist (available at https://dx.doi. org/10.21037/atm-21-3104).

\section{Methods}

\section{Ethical compliance}

All procedures performed in this study involving human participants were in accordance with the Declaration of Helsinki (as revised in 2013). The study was approved by the ethics committee at the First Affiliated Hospital of Gannan Medical University (No. 201713104150) and informed consent was taken from all the patients.

\section{Subjects and DNA preparation}

The proband patient first presented to the hospital complaining of acute chest pain and was subsequently diagnosed with a thoracic aortic dissection according to a computed tomography angiogram (CTA). She was then diagnosed with MFS according to the 2010 revised Ghent nosology criteria (6). The clinical phenotype of her first- degree relatives was also investigated. After the relevant consent forms were signed, peripheral blood samples were collected from these relatives, and genomic DNA samples were isolated.

\section{Next-generation DNA sequencing and variant calling}

In light of the characteristic clinical features of MFS, the blood samples of the patient's relatives were screened for the 13 genes associated with hereditary thoracic aortic disease (ACTA2, COL3A1, FBN1, FBN2, MYH11, MYLK, PLOD1, SLC2A10, SMAD3, TGFBR1, TGFBR2, EFEMP2, and ELN). This gene panel screening was carried out by using a next-generation sequencing (NGS) tool (Realomics Inc., Shenzhen, China). The average sequencing depth was $150 \mathrm{x}$, and qualified sequence reads were arrayed to the human reference genome (NCBI GRCh37) using the Burrows-Wheeler Aligner (version 0.5.17; http://bio-bwa.sourceforge.net/) (7). SAMtools (version 0.1.18, http://samtools.sourceforge.net/). Picard (http://picard.sourceforge.net/) and GATK (http://www. broadinstitute.org/gsa/wiki/index.php/Home_Page) were used for removing duplicate reads, alignments, and recalibrations. Potential single-nucleotide variants (SNVs) and small insertions and deletions (indel) were called and filtered using GATK3.7. High-confidence SNV and indel variants were then noted using snpEff (Version 4.2; http://snpeff.sourceforge.net/), and all variants were annotated according to the control population of the 1000 Genomes Project (2014 October release, http:// www.1000genomes.org), ExAC (http://exac.broadinstitute. org), EVS (http://evs.gs.washington.edu/EVS), the disease databases of ClinVar (http://www.ncbi.nlm.nih.gov/clinvar), and Online Mendelian Inheritance in Man (OMIM; http://www.omim.org).

\section{Statistical analysis}

Considering MFS is a rare Mendelian disorder, we calculated the frequency of each variant in gene targets in the proband by using a large control dataset from 2504 individuals in the 1000 Genomes Project phase 5 dataset, which contains data from individuals from five "superpopulations" (European, African, East Asian, South Asian, and ad-mixed American). We repeated the analysis for variants filtered according to minor allele frequency (MAF) thresholds $<0.05 \%$. We then filtered rare variants 
according to two scenarios protein-altering variants and loss-of- function (LOF) variants. The interpretation of the pathogenicity of all genetic variants was undertaken following the guidelines of the American College of Medical Genetics and Genomics (8). In the genomic DNA of the patient, we classified a frameshift variant of the FBN1 gene as "pathogenic/likely pathogenic" due to multiple lines of pathogenic evidence. According to the above guidelines, this result details when a variant has a greater than $90 \%$ chance of being disease-causing. Mutationtaster2 was used (9) to further determine whether the putative functions of the mutations were disease causing.

\section{Sanger sequencing}

The frameshift variant of FBN1 (NM_000138.4) was confirmed by Sanger sequencing. The targeted fragment was amplified by polymerase chain reaction (PCR) using a primer pair (GCATCACCAACCCTCCAATC; TGCTGAGTCTACAAGTCTGGT). PCR products were purified and sequenced by Tianyi Huiyuan Inc. (Guangzhou, China).

\section{Results}

\section{Family pedigree and patient information}

The proband (II-1), a 39-year-old female, was admitted to the hospital due to acute chest pain and was diagnosed with Standford B aortic dissection according to CTA (Figure 1A). She was then treated with an endovascular stent graft that spanned from the aortic arch to the descending aorta. In addition to the patient's aortic dissection, other clinical features of MFS were identified. These included the dilation of her carotid and pulmonary artery, as well as multiple skeletal disorders as described in Table 1. Her mother resembled the proband in looks and died at age 32 due to acute chest pain caused by aortic dissection. In accordance with the Ghent nosology criteria, the proband (II-1) was diagnosed as MFS because his features met the criteria that is a systemic score $>7$ and family history. The patient's 14-year-old son also displayed a dilated aortic bulb at the descending aorta next to the aortic arch, the same rupture site as that of the patient's dissection (Figure 1B), consequently was diagnosed MFS syndrome based on his family history and clinical phenotype.

\section{Sequencing analysis}

Given the high-mortality risk of the FBN1 variant, we performed genetic counseling and next-generation gene panel sequencing. Bioinformatic analysis revealed a novel FBN1 frameshift variant c.5081_5082insT (p. Leu1694Phefs ${ }^{*}$ ) in exon 42 (chr15:48755421), which was further confirmed by Sanger sequencing (Figure 1C,1D). The heterozygous peak of Sanger sequencing for the 2 patients demonstrated that the indel mutation was not yet present in the unaffected relative (Figure 1C). The insertion variant was further confirmed using clone-based sequencing (Figure 1D). We also found that the mutation c.5081_5082insT did not exist in known databases (UMDFBN1 (http://www.umd.be/), ClinVar (https://www.ncbi. nlm.nih.gov/clinvar/), UCSC common SNP (http:// genome.ucsc.edu/), dbSNP (http://www.bioinfo.org.cn/ relative/dbSNP\%20Home\%20Page.htm), and the 1000 Genomes Project) or in published articles. These results reveal that the proband (II-1) passed this FBN1 variant to her son (III-1) and that the genotype is segregated by phenotype in this family.

Further analysis indicated that this insertion caused a change in amino acids 1694 to 1701 and a deletion of large fragments in amino acids 1702 to 2871 (Figure 1D). Compared to the wild-type gene, this truncated protein lacks 23 exons in the $\mathrm{C}$-terminus, corresponding to 2 TGF- $\beta$ domains, 18 cbEGF-like domains, and 1 fibulinlike domain (Figure 1E). We further determined that this gene variation likely affects protein function and may subsequently result in disease (as predicted by the MutationTaster2). In accordance with standards and guidelines for the interpretation of sequence variants developed by the American College of Medical Genetics and Genomics, c.5081_5082insT can be considered as a pathogenic variant of this MFS family accompanied with early-onset familial thoracic aortic aneurysms (8). Considering that MFS's hereditary fashion, we also genotyped her little son at the pathogenic locus using Sanger sequencing and consequently excluded him as an MFS patient.

\section{Discussion}

The genotype-phenotype correlation was recognized as an important factor not only in diagnosis but also in the 
A

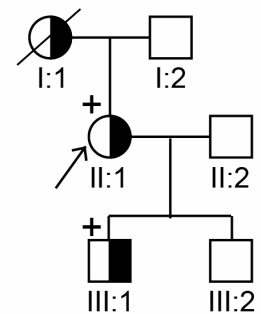

+ Heterozygous c.5081_5082insT FBN1 mutation

Thoracic aortic aneurym and/ or dissection

C

II: 1, III:1

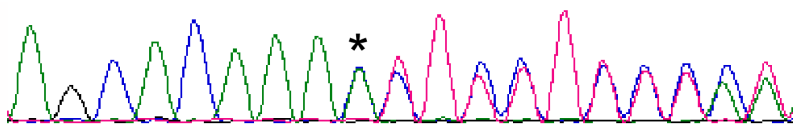

B
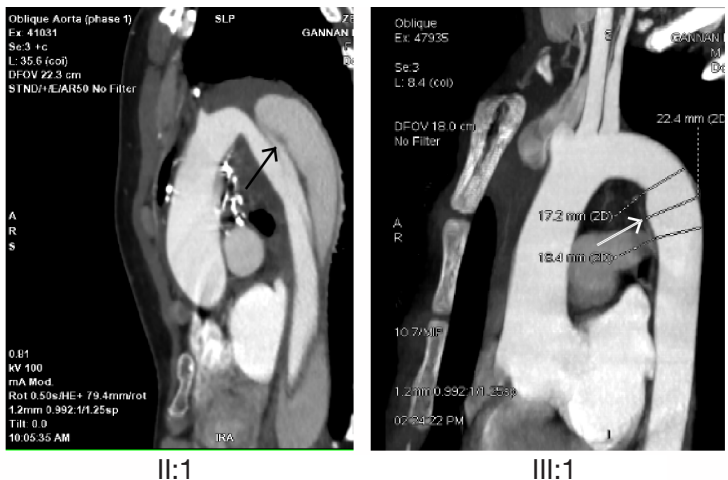

III:2

II:1, III:1

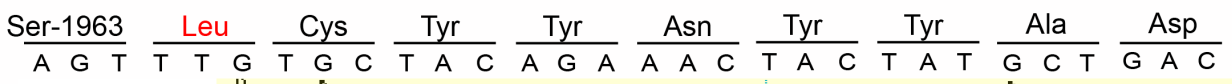

Wild type

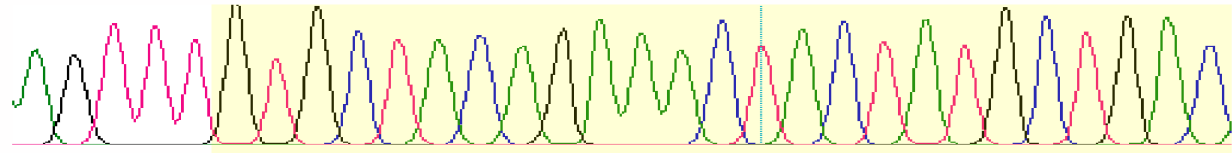

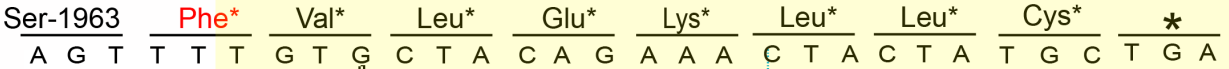

c.5081_5082insT

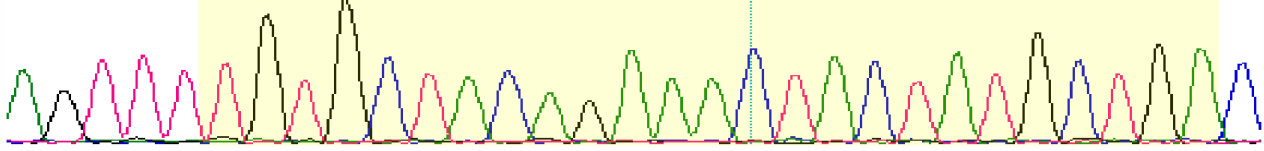

E

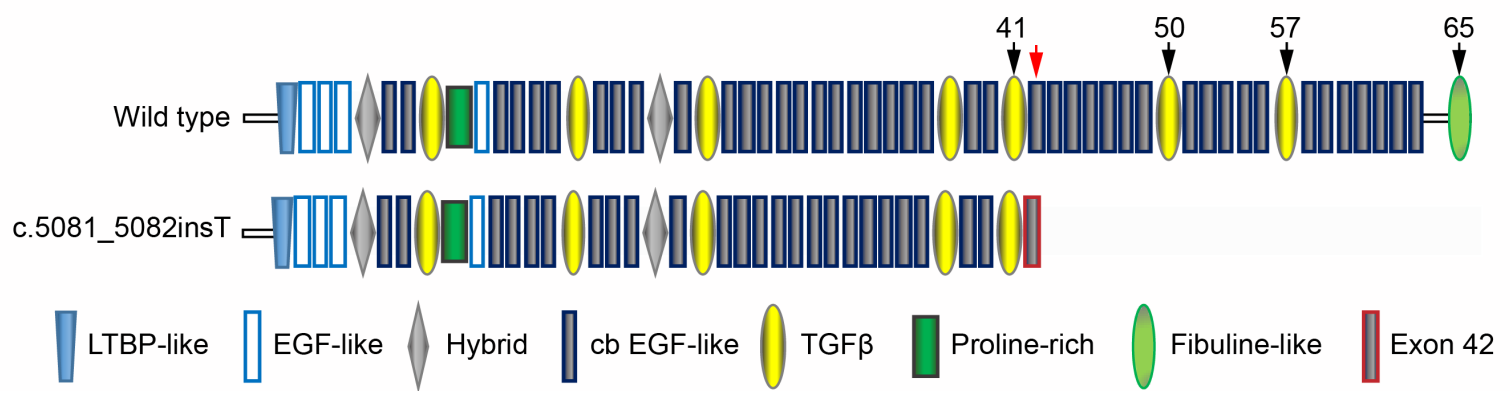

Figure 1 Family pedigree and molecular characterization of the variant c.5081_5082insT in FBN1. (A) Family pedigree. The individuals affected (I:1, II:1, III:1) are indicated by black squares and circles, and the proband is indicated by an arrow. (B) Thoracic aortic features of the proband (II:1) and her son (III:1). (C) The heterozygous variant c.5081_5082insT in exon 42 of FBN1. Sanger sequencing showed that the insertion was carried by II:1 and III:1 (indicated by the asterisk) and the corresponding normal sequence in unaffected family members. (D) Sequencing analysis of DNA clones from 2 affected individuals who had the heterozygous c.5081_5082insT variant. The upper tracer is the wild-type clone sequence, the lower tracer is the mutant clone sequence with the variant c.5081_5082insT. (E) The mutation identified in FBN1. Exon encoding and the different domains are numbered in bold, and the insertion is in red. The upper tracer is the integral FBN1, and the lower tracer is the truncated protein. cb-EGF, calcium-binding epidermal growth factor; EGF, epidermal growth factor; $F B N 1$, gene encoding fibrillin-1; LTBP, latent TGF- $\beta$ binding protein; TGF- $\beta$, transforming growth factor $\beta$. 
Table 1 The clinical features of the family as detailed in the Ghent nosology criteria

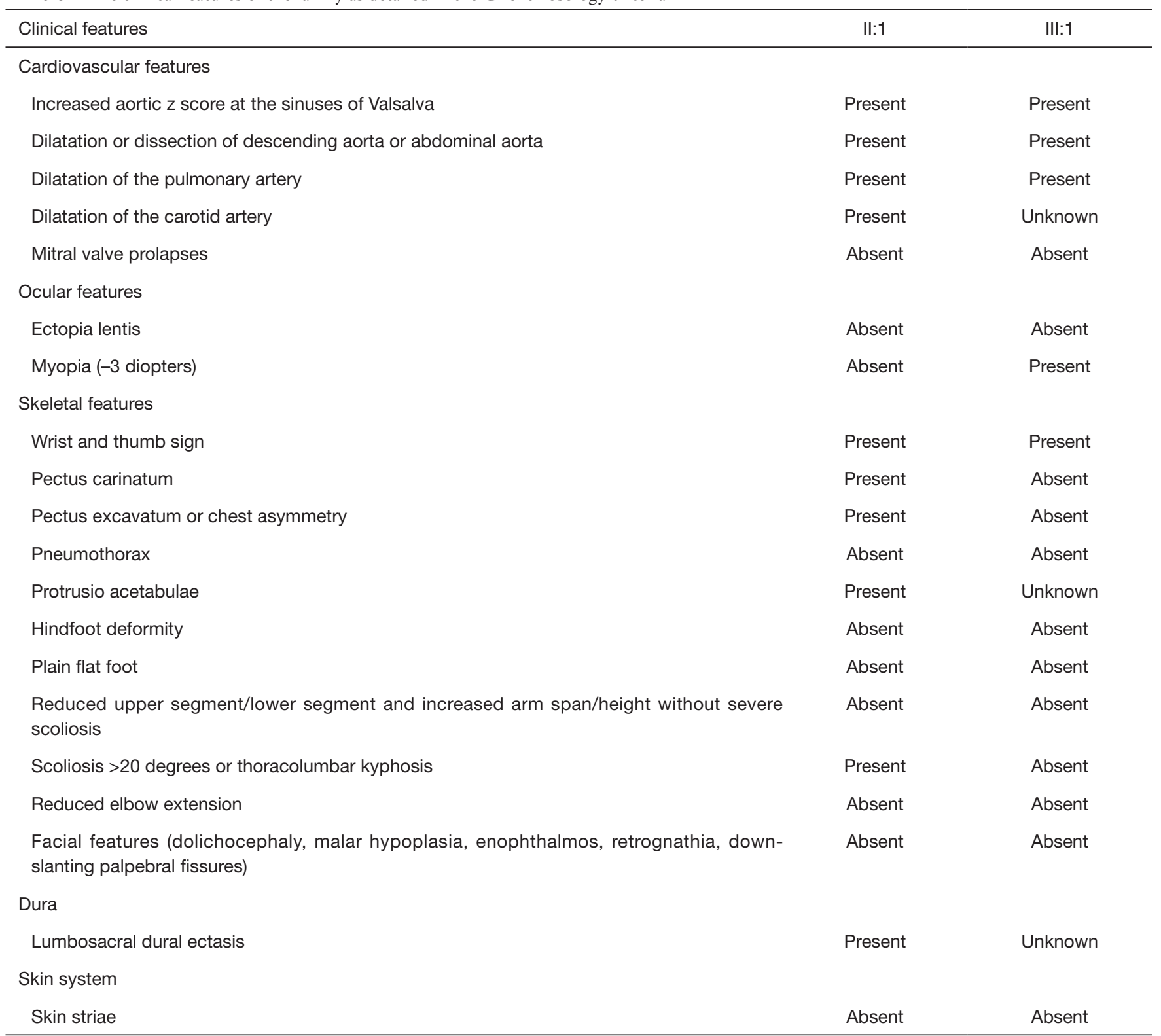

risk stratification and clinical management, of patients suspected to suffer from MFS (10). As MFS based purely on the mutation type is challenging, establishing a better genotype-phenotype correlation may prove to be an important step in the future treatment of MFS. In this case study of a Chinese family, we discovered that the novel insertion mutation c.5081_5082ins T (p. Leu1694Phefs*9) is associated with MFS characterized by early-onset familial TAA and accompanying multiple skeletal defects. The presence of the premature termination codon truncates
FBN1 protein, causes an haploinsufficiency (HI)-inducing effect, and consequently reduces the amount of functional FBN1.

The mutations that take place in $F B N 1$ have been classified into different categories including missense, which has a so-called dominant negative (DN) effect, and frameshift, nonsense and splice, all of which have a HI effect (11). A few studies have observed a trend in the association between genotype and clinical outcomes. For example, in a multicenter clinical trial, Faivre 
et al. evaluated the effect of mutation type and location, concluding that MFS patients with truncating variants in FBN1 have a more severe skeletal and skin phenotype compared with MFS patients with missense mutations (2). Moreover, several single-center prospective cohort studies have analyzed the correlation between aortic events and mutation classifications in MFS patients, suggesting that those diagnosed with truncating variants in the $F B N 1$ gene have a higher chance of experiencing an aortic event $(10,12)$. Recently, Arnaud et al. further found that those truncating mutants not only experienced the high risk of aortic events but also consequently had a shorter life expectancy, compared with MFS patients with in-frame variants (13). Otherwise, Baudhin et al. observed that $48 \%$ of MFS patients with a truncating, or splicing variant did not report an aortic event (12). Unlike truncating mutations, the number of missense mutations were enough to establish the correlation between MFS severity and mutation location. The missense mutations in exons 24-32 had been proven consistently by several studies that these variants resulted in early onset aortic risk and even severe forms of MFS through a dominant negative effect (2). However, in this region, truncating variants were not associated with aortic risk (13). These findings suggest that further research into the location of the pathogenic truncating variants of individuals with MFS may have significant implications for understanding the severity of the disease.

In this present study, the FBN1 variant, c.5081_5082insT, was noted to cause a truncated C-terminus, resulting in a lack of 1169 amino acids translated by exons $42-65$. The 2 patients diagnosed with the variant also fulfilled the criteria for skeletal and cardiovascular manifestation, but notably showed no ocular or skin anomalies. They both also had a positive wrist and thumb sign, with only the proband experiencing pectus carinatum, chest asymmetry, and scoliosis (Figure S1). What is also important to note is that the proband presented thoracic aortic dissection at 39 years of age, her mother died from aortic dissection at age 32, and her 14-year-old son has been identified as having an aortic sinus dilatation and a dilated aortic bulb at the descending aorta next to the aortic arch. This indicates that the c.5081_5082insT mutation caused by MFS has resulted in the patient's son having a high-mortality risk, as well as a high risk of experiencing some form of aortic complication, which in the future may require surgery. Our study also identified that the patient's son displayed some features of MFS which have not yet been defined as MFS in the Ghent nosology criteria, indicating the clinical values of etiological diagnosis and genetic counseling.

Ultimately, this research sheds further light on the important influence exons 42-65 have on aortic development. A previous report has described those patients with a mutation located in the 3 ' region (exon 59-63) of FBN1 were associated with a mild phenotype characterized by a lack of significant aortic pathology (14). The fragment from exons 44-49 have also been proven to regulate the bioavailability of TGF- $\beta$ and consequently contribute to Marfan pathogenesis (15). This has allowed us to recognize the important role TGF- $\beta$ signaling plays in aortic aneurysm progression (16). Losartan, a TGF- $\beta$ inhibitor, has also been noted to be particularly effective at reducing aortic root dilatation in MFS patients with HI FBN1 mutations as opposed to those with from DN mutations (17). Xu et al. reported that there was a significantly higher frequency of aortic dissection than aortic aneurysm in Marfan syndrome patients carried FBN1 frameshift and nonsense mutations, otherwise the in-frame mutants experienced the opposite tendency (18). Besides, the truncating mutations of FBN1 also contribute to non-syndromic sporadic or familial aortic aneurysms and dissections (19). Considering the severity of aortic involvement in truncating mutants, the location of truncating mutations in FBN1 associated with aortic events might enrich our knowledge on clinical management.

\section{Conclusions}

Through this study we have identified and confirmed a novel truncating mutation, c.5081_5082insT (p. Leu1694fs*9) in the FBN1 gene. This MFS mutation is characterized by early-onset familial TAA and is accompanied by variable skeletal manifestations. These findings make a significant contribution to research on the treatment and management of MFS patients with truncating variants in exons 42 to 65 .

\section{Acknowledgments}

Funding: This work was financially supported by the National Natural Science Foundation of China (No. 31671288), the Innovation Team Foundation (No. TD201902), and the Science and Technology Project of Jiangxi Health Committee (No. 202130676).

\section{Footnote}

Reporting Checklist: The authors have completed the MDAR reporting checklist. Available at https://dx.doi. 
org/10.21037/atm-21-3104

Data Sharing Statement: Available at https://dx.doi. org/10.21037/atm-21-3104

Conflicts of Interest: All authors have completed the ICMJE uniform disclosure form (available at https://dx.doi. org/10.21037/atm-21-3104). The authors have no conflicts of interest to declare.

Ethical Statement: The authors are accountable for all aspects of the work in ensuring that questions related to the accuracy or integrity of any part of the work are appropriately investigated and resolved. All procedures performed in this study involving human participants were in accordance with the Declaration of Helsinki (as revised in 2013). The study was approved by the ethics committee at the First Affiliated Hospital of Gannan Medical University (No. 201713104150) and informed consent was taken from all the patients.

Open Access Statement: This is an Open Access article distributed in accordance with the Creative Commons Attribution-NonCommercial-NoDerivs 4.0 International License (CC BY-NC-ND 4.0), which permits the noncommercial replication and distribution of the article with the strict proviso that no changes or edits are made and the original work is properly cited (including links to both the formal publication through the relevant DOI and the license). See: https://creativecommons.org/licenses/by-nc-nd/4.0/.

\section{References}

1. Franken R, Teixido-Tura G, Brion M, et al. Relationship between fibrillin-1 genotype and severity of cardiovascular involvement in Marfan syndrome. Heart 2017;103:1795-9.

2. Faivre L, Collod-Beroud G, Loeys BL, et al. Effect of mutation type and location on clinical outcome in 1,013 probands with Marfan syndrome or related phenotypes and FBN1 mutations: an international study. Am J Hum Genet 2007;81:454-66.

3. De Backer J, Loeys B, Leroy B, et al. Utility of molecular analyses in the exploration of extreme intrafamilial variability in the Marfan syndrome. Clin Genet 2007;72:188-98.

4. Sakai LY, Keene DR, Renard M, et al. FBN1: The diseasecausing gene for Marfan syndrome and other genetic disorders. Gene 2016;591:279-91.
5. Verstraeten A, Alaerts M, Van Laer L, et al. Marfan Syndrome and Related Disorders: 25 Years of Gene Discovery. Hum Mutat 2016;37:524-31.

6. Loeys BL, Dietz HC, Braverman AC, et al. The revised Ghent nosology for the Marfan syndrome. J Med Genet 2010;47:476-85.

7. Li H, Handsaker B, Wysoker A, et al. The Sequence Alignment/Map format and SAMtools. Bioinformatics 2009;25:2078-9.

8. Richards S, Aziz N, Bale S, et al. Standards and guidelines for the interpretation of sequence variants: a joint consensus recommendation of the American College of Medical Genetics and Genomics and the Association for Molecular Pathology. Genet Med 2015;17:405-24.

9. Schwarz JM, Cooper DN, Schuelke M, et al. MutationTaster2: mutation prediction for the deepsequencing age. Nat Methods 2014;11:361-2.

10. Wang Y, Lian Z, Zhou Y, et al. Differential diagnosis of Marfan syndrome based on ocular biologic parameters. Ann Transl Med 2020;8:1354.

11. Dietz HC. Potential Phenotype-Genotype Correlation in Marfan Syndrome: When Less is More? Circ Cardiovasc Genet 2015;8:256-60.

12. Baudhuin LM, Kotzer KE, Lagerstedt SA. Increased frequency of FBN1 truncating and splicing variants in Marfan syndrome patients with aortic events. Genet Med 2015;17:177-87.

13. Arnaud P, Milleron O, Hanna N, et al. Clinical relevance of genotype-phenotype correlations beyond vascular events in a cohort study of 1500 Marfan syndrome patients with FBN1 pathogenic variants. Genet Med 2021;23:1296-304.

14. Palz M, Tiecke F, Booms P, et al. Clustering of mutations associated with mild Marfan-like phenotypes in the $3^{\prime}$ region of FBN1 suggests a potential genotype-phenotype correlation. Am J Med Genet 2000;91:212-21.

15. Chaudhry SS, Cain SA, Morgan A, et al. Fibrillin-1 regulates the bioavailability of TGFbeta1. J Cell Biol 2007;176:355-67.

16. Holm TM, Habashi JP, Doyle JJ, et al. Noncanonical TGF $\beta$ signaling contributes to aortic aneurysm progression in Marfan syndrome mice. Science 2011;332:358-61.

17. Franken R, den Hartog AW, Radonic T, et al. Beneficial Outcome of Losartan Therapy Depends on Type of FBN1 Mutation in Marfan Syndrome. Circ Cardiovasc Genet 2015;8:383-8.

18. Xu S, Li L, Fu Y, et al. Increased frequency of FBN1 frameshift and nonsense mutations in Marfan syndrome patients with aortic dissection. Mol Genet Genomic Med 
Page 8 of 8

2020;8:e1041.

19. Tan L, Li Z, Zhou C, et al. FBN1 mutations largely contribute to sporadic non-syndromic aortic dissection.

Cite this article as: Duan $\mathrm{Y}$, Chang H, Ling J, Liu S, Zhong Y. A novel variant in fibrillin-1 is responsible for earlyonset familial thoracic aortic aneurysms in Marfan patients. Ann Transl Med 2021;9(15):1240. doi: 10.21037/atm-21-3104

\section{Duan et al. A novel variant for familial thoracic aortic aneurysms}

Hum Mol Genet 2017;26:4814-22.

(English Language Editors: J. Goetz and J. Gray) 

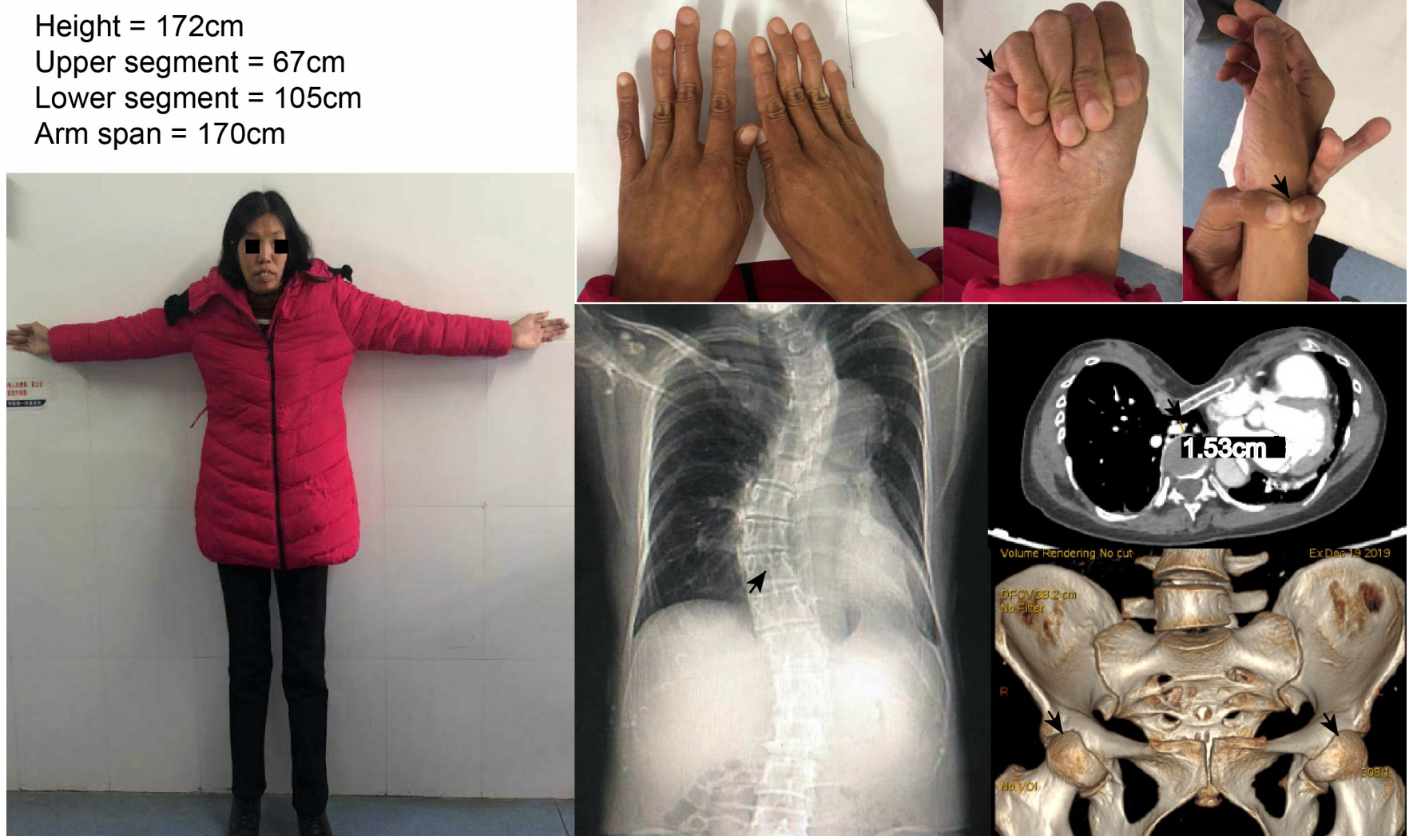

Figure S1 Skeletal features of the proband: note the wrist and thumb sign, pectus carinatum, and mild scoliosis. 